$\Rightarrow$ IMMUNOTHERAPY

\section{TNFR superfamily trimers}

Signalling through receptors of the tumour-necrosis factor receptor (TNFR) superfamily requires the formation of trimeric ligand complexes that activate receptor chains and downstream events. The structures of several TNF family members in complex with their cognate receptors reveal that each trimeric ligand complex interacts with three monomeric receptor chains. Papers published in Nature Chemical Biology and Science describe a new approach to targeting TNFR pathways using small molecules to agonize and antagonize members of the TNFR superfamily, respectively.

The TNFR superfamily includes receptors such as CD40, lymphotoxin receptor and nerve growth factor receptor. These receptors are involved in a wide range of diseases from inflammatory conditions to cancer. Inhibition of TNF $\alpha$ by the biologics etanercept (Enbrel; Amgen), infliximab (Remicade; Centocor/ Schering-Plough) and adalimumab (Humira; Abbott) for the treatment of rheumatoid arthritis has established itself as an effective therapeutic strategy, and has validated the targeting of this pathway. Current strategies for the development of small-molecule antagonists of TNF $\alpha$ and its signalling pathway include inhibiting TNFconverting enzyme or other upstream signal transduction molecules such as nuclear factor- $\kappa \mathrm{B}$. However, developing functional small-molecule agonists and antagonists of these receptors is challenging because of the requirement to disrupt protein-protein interactions as well as integrating the correct three-fold symmetry.
Guichard and colleagues designed small synthetic molecules with threefold symmetry that can mimic CD40 ligand (CD40L) homotrimers. The molecular scaffold incorporated a circular core with three arms, at twelve, four and eight o'clock positions. To each arm, they attached a short peptide derived from CD40L that is known to interact with CD40. The geometry and distances of the $\mathrm{C}_{3}$ molecule could match those of the CD40L homotrimer and bind to and activate CD40. The authors demonstrated that the molecules mimic the action of CD40L by signalling through CD40 in B lymphoma cells and dendritic cells. Furthermore, the molecules targeted only CD40 and did not activate other TNF receptor proteins. This specificity suggests that these $\mathrm{C}_{3}$ molecular cores could be a versatile platform for targeting different TNFRs by decorating them with the appropriate peptide fragments.

Cunningham and colleagues identified a small-molecule inhibitor of TNF $\alpha$ that promotes disassembly

\title{
In vitro patterns predict clinical effects
}

Accurate prediction of the clinical effects of compounds in preclinical development would considerably improve the efficiency of drug discovery and reduce costly late-stage failures. Writing in Nature Chemical Biology, Fliri and colleagues show that the side effects of known drugs can be linked to their effects in in vitro protein-inhibition assays, providing a potential strategy for predicting the clinical effects of novel compounds early in preclinical testing. The current study builds on earlier work by the same group in which they analysed the in vitro interactions of 1,567 structurally diverse compounds with a panel of 92 proteins representative of the 'druggable' proteome. Compounds were screened at a single high concentration in ligand binding assays so that the percent inhibition values obtained provided an indication of the potential of each compound to interact not just with the proteins in the panel, but with those in the same family.

These percent inhibition values were then translated into biological activity spectra - biospectra - that express the probability of a particular compound inducing a certain pattern of proteinnetwork perturbations. Comparison of the biospectra of different compounds showed that compounds with similar structures and pharmacological effects clustered together, and that biospectra of novel compounds could be predicted from their molecular structure.

Encouraged by these results, the authors set out to investigate how predictive biospectra are of the clinical effects of drugs. First, using information from the labels of 1,045 drugs, the authors derived 'side-effect spectra' based on the classification of the drugs according to 591 types of side effect that they can show, such as nausea. Analysis of these spectra revealed that compounds with similar structures or protein-network perturbations clustered with each other.

Then, taking a subset of the drugs for which the data necessary to

create biospectra were available, Fliri et al. demonstrated that there was a meaningful relationship between the way that drugs clustered according to their biospectra and the way that they clustered according to their side-effect spectra. So, this approach provides a mechanism for linking the molecular structure of compounds, their properties in simple biological assays and their clinical effects.

The key to improving the quality of predictions of the clinical effects of novel compounds from their structure and effects in in vitro assays will be to improve the amount and quality of data on known compounds used in the method. So, as the authors highlight, the potential to reduce the risk of failure in clinical development using this method could provide incentives to create public databases containing preclinical, clinical and safety information not just on marketed medicines but on those that fail in clinical trials.

Peter Kirkpatrick

ORIGINAL RESEARCH PAPER Fliri, A. F. et al.

Analysis of drug-induced effect patterns linking structure and side effects of medicines. Nature Chem. Biol. 1, 389-397 (2005)

FURTHER READING Fliri, A. F. et al. Biological spectra analysis: linking biological activity profiles to molecular structure. Proc. Natl Acad. Sci. USA 102, 261-266 (2005) | Fliri, A. F. et al. Biospectra analysis: model proteome characterizations for linking molecular structure and biological response. J. Med. Chem. 48, 6918-6925 (2005) 
of the TNF $\alpha$ trimeric complex, and it is able to inhibit TNF $\alpha$ activity in biochemical and HeLa cell-based assays. The compound is composed of trifluoromethylphenyl indole and dimethyl chromone moieties linked by a spacer. X-ray crystallography revealed that the single-compound molecule displaces a subunit of the TNF $\alpha$ trimer to form a complex with a TNF $\alpha$ dimer. The authors considered how the compound acts to cause formation of the dimer; experimental evidence suggests that the compound actively interacts with the trimer to form an intermediate complex that undergoes accelerated subunit dissociation. This work suggests that small molecules can disrupt tightly pre-associated oligomeric proteins.

Melanie Brazil

ORIGINAL RESEARCH PAPER Fournel, S. et al. C3-symmetric peptide scaffolds are functional mimetics of trimeric CD40L. Nature Chem. Biol. doi:10.1038/nchem bio746 (2005) | He, M. M. et al. Small-molecule inhibition of TNF- $\alpha$. Science $\mathbf{3 1 0}$, 1022-1025 (2005)

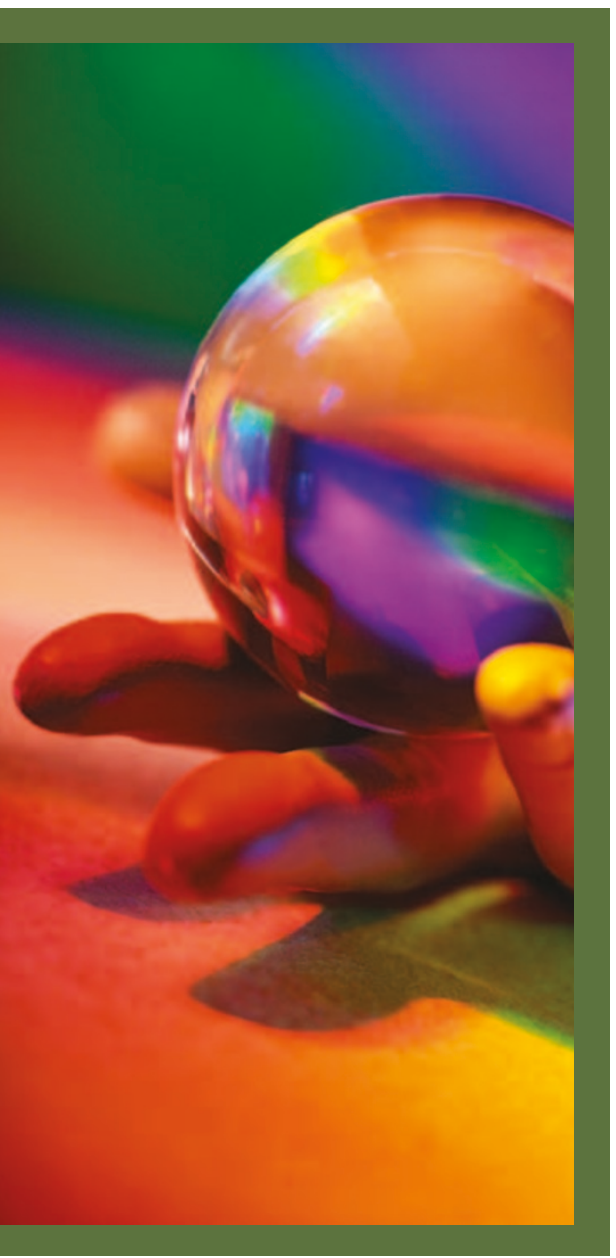

\section{Know thy enemy}

In the battle against cancer, knowing what you're up against is crucial. Two recent Nature papers provide elegant examples of how genetically characterizing tumours can help to predict progression and drug sensitivity, and show that patient stratification will be essential to evaluate the efficacy of targeted anticancer drugs.

The use of 'gene signatures' - specific geneexpression profiles for particular tumour types is well established in cancer diagnosis, target discovery and drug screening. Joseph Nevins and colleagues took a fresh approach to this concept and generated signatures that represent the activation status of individual oncogenic pathways. They used recombinant adenoviruses to express various oncogenic signals in quiescent human mammary epithelial cells (HMECs) so that the subsequent gene-expression signature represents the downstream effects of that particular oncogenic event. Principal components analysis was then used to select a set of genes that represents the signature for that pathway.

The authors successfully used the signatures to distinguish between cells expressing each oncogenic activity and the control cells, both in HMECs and in tumour samples derived from standard mouse cancer models. In non-small-cell lung carcinoma samples, they found that a Ras pathway signature distinguished lung adenocarcinoma from lung squamous cell carcinoma, confirming that the former has a higher probability of Ras mutation than the latter.

However, real predictive power was achieved by combining pathway signatures. Analysis of lung tumours showed that adenocarcinomas predicted to have low-level Ras activity generally also had elevated Myc, E2F3, $\beta$-catenin and Src activity. Integrating these signatures identified a patient subpopulation with poor survival. Perhaps most importantly, the authors could also predict drug sensitivity: growth inhibition measured in cancer cell lines exposed to Ras or Src inhibitors correlated with predicted status of the corresponding pathway, indicating the potential of these signatures as a guide to therapy in patients.

Genetic characterization of tumours was also used by Neal Rosen et al. in a study of the effects of Braf and Raf mutations on the mitogen-activated protein kinase kinase (MEK) and extracellular signal-related kinase (ERK) pathway. Mutations in Ras and Braf are often mutually exclusive and therefore might exert their oncogenic activity through common downstream signals that could be exploited as drug targets.

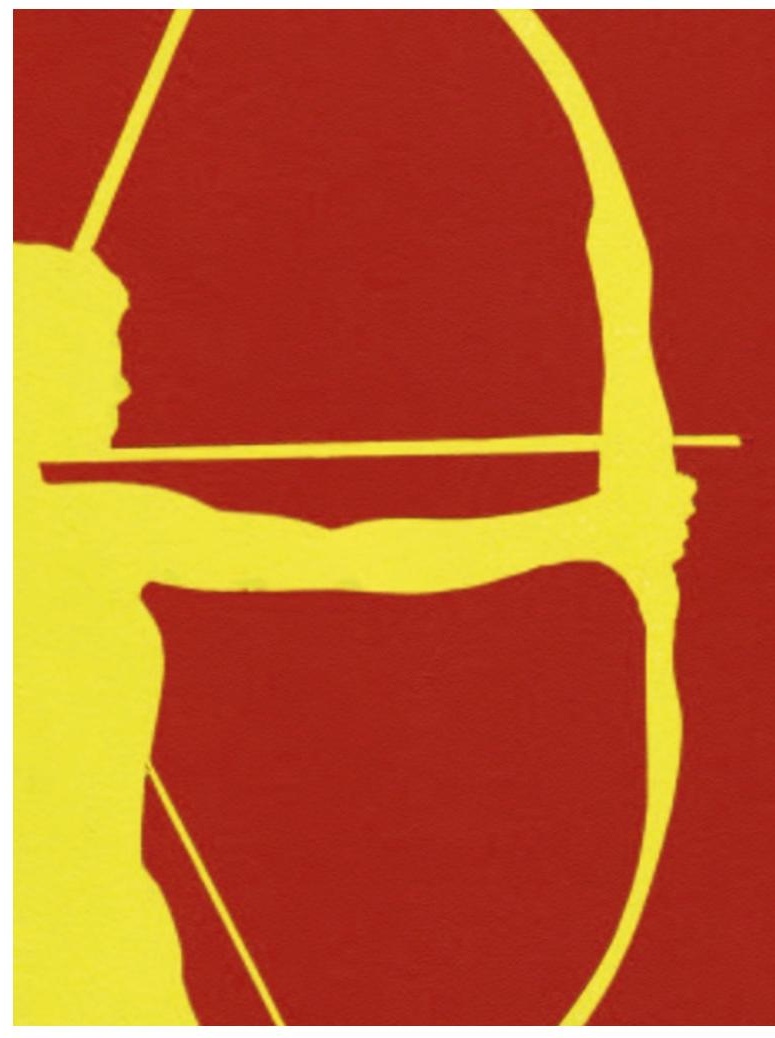

The authors used a selective MEK inhibitor in cells with Ras or Braf mutations and found that tumours with Braf mutations were exquisitely sensitive to MEK inhibition compared with Ras mutant and wild-type cells. Indeed, using the National Cancer Institute's chemical sensitivity database ( $\mathrm{NCl} 60$ ), the authors ranked compounds according to growth inhibition of tumour cells with Braf mutations and found that the most effective compounds were all known to inhibit MEK. Furthermore, in mice bearing wild-type and Braf-mutant xenograft tumours, growth of the Braf-mutant tumours was completely suppressed.

The finding that tumours containing mutations in Braf are much more dependent on MEK inhibition than tumours with mutant Ras has several implications for drug development. First, MEK inhibitors have a selectivity that could be exploited in the treatment of Braf-mutationdependent cancers; however, in certain genetic contexts, MEK inhibitors might need to be used in combination with other targeted therapies. Second, the authors speculate that their results could explain the mixed success of Braf inhibitors in clinical trials, and that patients should be stratified according to the mutation status of their tumours in future clinical studies.

\section{Joanna Owens}

ORIGINAL RESEARCH PAPERS Bild, A. H. et al. Oncogenic pathway signatures in human cancers as a guide to targeted therapies. Nature doi:10.1038/nature04296 (2005) | Solit, D. B. et al. BRAF mutation predicts sensitivity to MEK inhibition. Nature doi:10.1038/nature04304 (2005) 